\title{
A Cognitive Linguistic Study of Verb-copying Sentences in Mandarin Chinese
}

\author{
Yuting Xu \\ Faculty of English Language and Culture \\ Guangdong University of Foreign Studies, Guangzhou, China.
}

\begin{abstract}
This study concerns the verb-copying structure "S+V+Object+V+Resultative" in Chinese from the perspective of cognitive grammar. It views the construction as composited from component structure "S+V+Object" and "V+Resultative" and reveals the compositional path and mental representation of this construction. The results indicate that component SVO elaborates the schematic trajector of $\mathrm{V}$ in Component VR. This conceptual correspondence lays the foundation for the integration of component SVO and VR, which constitutes the internal motivation of SVOVR construction. Component SVO serves as the cognitive reference point for the conceptualization. The elements in the dominion of action that SVO designates are extracted as conceptualization target. SVOVR construction is organized in the pattern of Baseline and Elaboration. In local terms, each stratum provides the potential for the next and the conceptualization result - the linear structure - is completed in a cumulative fashion. In global terms, component SVO and VR serve as dual baseline whose mutual elaboration yields a composite structure of greater complexity. SVOVR construction imposes some restrictions on the repeated verb. The verb needs to designate the actions that can be repeated and continued. Structure One cannot have any tense or the aspect markers. SVOVR construction does not stand in isolation from other units in linguistic system; it is categorized by the conventionalized SVO structure and predicate-complement VR structure in Chinese language system, which is the external motivation for this construction.
\end{abstract}

Keywords: verb-copying sentence; reference point; Baseline and Elaboration; compositional path

\section{INTRODUCTION}

Verb-copying construction (VCC) is typical in Mandarin Chinese. It refers to a grammatical process in which a verb is "copied" after its direct object when in the presence of certain adverbial elements. Thus, we have a construction of the following form:

\section{(subject) verb direct object verb $\underline{\underline{\text { adverbial element }}}$}

What is labeled direct object can be either an actual direct object or the object component of a verbobject compound. The term adverbial element is meant to be a cover term for four different types of adverbial expressions including quantity adverbial phrase, complex stative construction, locative 
phrase and directional phrase [6]. This linguistic pattern was firstly noticed by a Chinese grammarian Li Wang who termed it narrative words repetition. In his Chinese Grammar Theory in 1944 [15], he proposes that the adverbial element must be adjacent to the verb. In another word, the following form is ungrammatical:

\section{*(subject) verb direct object adverbial element}

For example, wo chiwan fan le is grammatical while wo chifan wan le is not. Therefore, the verb and the adverbial elements is a unified whole. Even though the verb has direct object, the direct object cannot separate the verb from the adverbial element. Meanwhile, compound verb cannot be separated by the adverbial element. This conflict can be reconciled by repeating the verb without making any compromise. In Chinese Spoken Language, Yuenren Chao [19] has the similar argument that when both Verb + Object and Verb + Adverbial structures cannot be separated, the only thing we can do is to repeat the verb. Most Chinese scholars basically have the same viewpoints with Wang and Chao. That is, the verb-copying construction is used to resolve the dilemma of object and adverbial competing for the verb. The verb-copying construction has received much attention both home and abroad. Some scholars focused on its syntactic conversion with other syntactic structures like $b a /$ bei construction and S-P predicate construction ([5]; [11]; [8]; [3]; [17], etc.). Some scholars investigate this linguistic pattern from the perspective of information focus ([14]; [16]), pragmatics ([3]; [7]), discourse [10] and categorization ([8]; [13]). With the rise of cognitive linguistics, some scholars begin to investigate these linguistic structures from the perspective of human cognition. Dai ([1]; [2]) holds that verb copying is the imitation of continuous action and state and conceptual representation on the grammatical level. He argues that verb copying is motivated by the semantics and the event can be repeated and continued. The verb cannot be copied if the it designates an instantaneous action or change. Wang [14] investigates this linguistic pattern within the framework of construction grammar. He combines the Event-domain Cognitive Model with Multiple Inheritance Model and explains the mechanism behind the verb-copying construction. These studies have made great achievements in systematic description from the aspects of semantics, syntax and pragmatics. However, the schematicity and the generality of mental representations of VC construction have not been fully unearthed. This study aims to provide solutions for the existing problems and contribute to the growing body of research on cognitive study of syntax in Mandarain Chinese. This study will take the specific type of verb-copying structure "S + Verb + Object + Verb + Resultative" (short as SVOVR) as the object and investigate the cognitive motivation of this construction from the perspective of cognitive grammar. The research questions this study aims to address can be summarized as follows:

1. What are the basis for the integration of "SVO" and "VR"?

2. How are SVOVR construction mentally represented?

3. What are the restrictions for the verb in verb-copying construction?

\section{THEORETICAL FOUNDATION}

Cognitive grammar, referred to by the acronym CG in this paper, claims that language is an integral part of human cognition and takes seriously the goal of psychological reality in linguistic description. The psychological representation of a linguistic system is also referred to by linguists as grammar [21]. Unlike the orthodox linguistic paradigm where the syntactic structures are instructed by the rules and other operations yield the surface form, rules take the form of schemas which are patterns and regularities of any sort, at any level of specificity, reside in schematic units abstracted from occurring expressions in CG [22]. In CG, a language needs only three kinds of 
symbolic structures: semantic, phonological and symbolic. Semantic structures are conceptualizations exploited for linguistic purposes, notably as the meanings of expressions. The phonological structure includes not only sounds but also gestures and orthographic representations. A symbolic structure is bipolar: $\mathrm{S}$ is its semantic pole, and $\mathrm{P}$ is its phonological pole [22]. There is no strict division of lexicon, morphology and syntax. They form a continuum fully reducible to assemblies of symbolic structures. The central notion of CG is that grammatical structure is inherently symbolic. In CG, grammatical patterns are represented by means of schemas. A construction is defined as either an expression (of any size), or else a schema abstracted from expressions to capture their commonality (at any level of specificity) [22]. Expressions and the patterns they instantiate are all symbolic in nature, which only differ in degree of specificity.

In CG, describing the grammar of a language consists primarily of describing its constructions. There are four basic factors in the description of construction: correspondences, profiling, elaboration and constituency. Of the four descriptive factors to be considered, correspondences are perhaps the most fundamental. A construction is an assembly of symbolic structures (form-meaning pairings) linked by correspondence. They indicate how component and composite structures fit together in a coherent assembly. At the semantic pole, they specify the conceptual overlap between component structures, thus providing the basis for their integration [22]. The phonological pole is mostly ignored, the semantic structures under discussion represent just one pole of symbolic assemblies. It is typical in constructions for the composite semantic structure to profile the same entity as one of the component structures. The component structure that inherits profile to the composite structure is referred to as the profile determinant in CG. It is typical in a construction for one component structure to contain a schematic substructure which other component serves to elaborate, i.e. characterize in finer-grained detail. The schematic element elaborated by another component is called an elaboration site, or e-site for short.

In CG, a full, finite clause profiles a grounded instance of a process type. In the simplest case, this type is directly specified by a lexical verb. The grounded structure consists of more than just the verb. Minimally, it further includes the verb's "arguments": the nominals that specify its profiled participants. Since a process is conceptually dependent on its participants, a verb evokes them schematically as an inherent aspect of its meaning [22]. Thus, the verb is the head and the nominals are complements because they elaborate salient substructures of it. A verb's complements are not limited to the subject and object nominals that specify its focal participant. Often its meaning incorporates additional schematic entities that are sufficiently salient to function as elaboration sites. Among these are participants which happen not to be focused as trajector or landmark [22]. For example, in She sent him flowers, the agent and recipient are of focal prominence, making she the subject and him the object. The mover in this construction is clearly a central participant which is essential to the meaning of send. An elaborating nominal in this case is a complement. A verb's semantic structure can also incorporate a schematic relationship that functions as an e-site. The verb put profiles the action that the trajector moves the landmark and results the latter in a new location. Besides the subject and object, it therefore takes a locative expression as a complement that specifies where the object winds up as in She put the flowers in the vase. CG takes the position that grammar consists in a person's grasp of an inventory of established linguistic conventions which are referred to as "units". Importantly, the inventory is claimed to be structured in the sense that each unit participates in relations of various kinds to many other units. 
Baseline and elaboration is the latest theoretical development of CG. The notions baseline and elaboration pertain to asymmetries observable in any facet of language structure or its conceptual and phonological basis [23]. B/E organization is utterly ubiquitous in the broadest sense. In one way or another, the baseline (B) is already, in place, or under control. Its elaboration (E) can be characterized abstractly as a function mapping B onto the higher-level structure BE. E is a kind of cognitive processing which is dynamically described as an operation which consists of augmentation, adaptation, or additional processing activity. B has some kind of priority and is generally substantive than E as well. Naturally, an elaborated structure (BE) can function as baseline for further elaboration representing a higher level of B/E organization [23]. The levels are layered and dynamically evolved. Baseline, elaboration and strata are diagramed in Figure 1. The boxes indicate strata $(\mathrm{S})$.

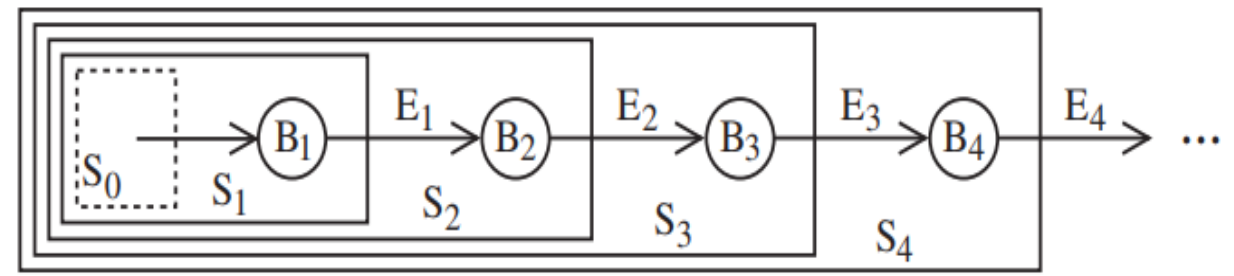

Figure 1. Baseline, elaboration, and strata

The key notion is that a structure does not just spring into existence ex nihilo, but always emerges from a substrate that supports it and creates the potential for its emergence. A given stratum, $S_{i}$, comprises an array of resources available for structure building; they include both mental capacities and the structures already in place.

\section{COMPOSITE STRUCTURE SVOVR}

A defining property of human language is the formation of complex structures out of simpler ones. In CG, Composite structure is a structure that results when two or more structures in a given domain (phonological, semantic, or symbolic) combine in a valence relation. In this study, SVOVR construction is defined as a composite structure of the two components SVO and VR with the same verb. VO and VR are component structures which integrate in a combinatory relationship (particularly a grammatical valence relation). In this section, we will discuss how the component structures VO and VR are integrated and fit together in a coherent way.

\section{Component structure of SVOVR construction SVO component}

Component SVO is canonical linguistic pattern in Chinese language. Inside this component structure, the verb's schematic trajector and landmark are elaborated by its focal participants which serve as subject and object of this syntactic structure, in a finer-grained way. In Chinese, verbs do not always appear as a single morpheme; there are a very large number of compound verbs like "paobu" running, "zoulu" walking and "shuijiao" sleeping etc. So, this component structure can be abstracted as SV with only the schematic trajector to be elaborated by the verb's focal participants in some circumstances.

In the composite structure SVOVR, the first component SVO is reified as a nominal expression and designates a specific event; its temporal profile has faded into the background while the second verb 
in component VR profiles relationship; that is, SVO component is in subordinate position of the composite structure despite the same verb with component VR. In English grammar, verbs should be non-finite, in infinitive or participle form, if they do not serve as predicate verbs in a sentence. The infinitive and participle form have corresponding morphological markers like to-, -ing and -ed. Unlike English, Chinese is not rich in morphological change. In Chinese grammar, a syntactic structure is a phrasal expression if it is contained within a more complex structure; it is realized as a sentence when used alone [9]. Therefore, the profile of Chinese verbal expression, things or relationship, can only be judged from the specific linguistic expressions. In SVOVR construction, the VO component as a whole is nominalized and profile a thing. VO cannot be negated by negative adverbials and reject tense and aspect markers. From the standpoint of usage event, this component is rarely used alone. When the addressee hears bare SVO expressions without any additional elements, they will expect another new information. For example, this bare SVO structure can appear in parallel form like "Wo change, ni tiaowu" I sing, you dance. Shen [12] named this kind of sentence as bound sentence and proposed that there are not only free and bound morphemes but also free and bound syntactic structures in Chinese language. The opposition between free and bound is also the opposition of boundedness and unboundedness in human cognition. The unbounded syntactic structure designates an action but not an event for it has no clear internal starting point and endpoint. In contrast, the bounded syntactic structure designates an event which has explicit starting and end points. Thus, component SVO is unbounded and dependent from the perspective of usage.

\section{VR component}

Component VR designates a processual relationship and bequeaths its profile to the composite structure as a whole. The schematic substructure of the verb in this component is not specified by its nominal participants; its schematic trajector is elaborated by component SVO which is nominalized and profiles a thing; its schematic landmark is elaborated by complement $\mathrm{R}$ which designates the results of the action designated by SVO. Negative adverbials, tense and aspect markers can be used in this component, which is different with component SVO; it is bounded in cognition. Besides, the semantics of complement R orients to the elements in the frame of SVO. Take a look at the following linguistic instantiations of SVOVR construction:

1. Wo zuofan zuo huai le guo

My cooking broke the pan.

2. Wo zoulu zou de jiao qi pao le

Walking made my feet blister.

3. Wo kanshu kan lei le

Reading made me tired.

4. Wo jiao meishu jiao chu jingyan le

Teaching art produced me some expertise.

In (1), the "guo" pan in R complement orients to instrument in the action of cooking. In (2), the "jiao" feet are indispensable parts of body involved in the action of walking. In (3), "lei" tired is the description of the physical state of the agent in the action of reading. In (4), the semantics of "jingyan" expertise orients towards the action of teaching itself. The designation of complement $\mathrm{R}$ is the resultative state of the elements in the action of component SVO. The action can result in the appearance of a new state or a new thing. In example (1) and (3), the action "zuofan" cooking and 
"kanshu" reading cause a new condition of the instrument "guo" pan and agent " $w o$ " I respectively; in example (2) and (4), the action "zoulu" walking and "jiao meishu" teaching art cause a new thing appear in the parts of body involved in the action and the action itself.

\section{Compositional path of SVOVR construction}

In CG, how an expression's composite meaning relates to those of its components (at successive levels of organization) is called its compositional path [22]. The conceptual correspondence motivates the integration of component SVO and VR. Integration depends on correspondences. For two semantic structures to combine syntagmatically, they must have some point of overlap; more precisely, a substructure of one is placed in correspondence with a substructure of the other, and these two substructures are construed as designating the same conceived entity. It is by virtue of having one or more such entities in common that two component structures can be integrated to form a coherent, more elaborate conceptualization [21].

At the lower level of organization, inside component SVO, the verb's schematic substructure is elaborated by its focal participants which serves as the subject and object of the verb. In SVOVR construction, SVO component as a whole profiles non-processual relationship and is reified as a nominal. The reified VO serves to elaborate the schematic trajector of the verb in VR. The verb in VR is conceptually dependent and profiles temporal relationship. Its schematic landmark is elaborated by R complement which designate the resultative state of the elements in the frame of SVO. The schematic trajector of this verb is elaborated by SVO component which designates the cause of the resultative state. Rather than seeing a composite structure as an edifice constructed out of smaller components, we can treat it as a coherent structure in its own right: component structures are not the building blocks out of which it is assembled, but function instead to motivate various aspects of it [21]. The composite structure inherits the profile of VR. Linguistic phenomena lend themselves more easily to a claim of partial rather than full compositionality [21]. The compositional path of SVOVR construction is demonstrated as Figure 2. Component SVO is reified and profiles complex non-processual relationship. Component VR designates a processual relationship. The schematic $\mathrm{TR}_{2}$ is elaborated by component SVO. The dotted line indicates correspondence and solid arrow leads from e-site to its elaboration. Component SVO designates a relationship which does develop through time but is non-processual by virtue of being viewed holistically, so that its temporal evolution is backgrounded. The bar along the time arrow in component VR indicates that its evolution through time is focused rather than backgrounded. At higher levels of organization, the composite structure inherits the profile of component VR and designates a processual relationship. Component SVO is viewed holistically in the composite structure and profiles a thing which is indicated by a surrounded ellipse. The component structures are not invoked for their own sake, but as "stepping-stones" for purpose of "reaching" the composite conception. 


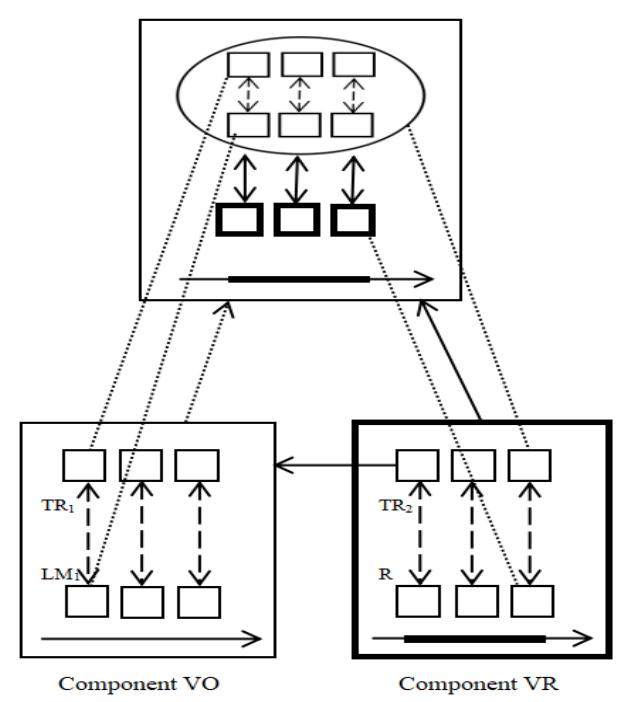

Figure 2. Compositional path of VOVR construction

Take one of the instantiations of the constructional schema, Wo zuofan zuo huai le guo, as an example. Its compositional path is as Figure 3. The composition of the component structures is motivated by the overlapping elements in their semantic structures. The components contribute asymmetrically to the target structure at the higher level of organization. The component structure zuo huai le guo bequeaths its profile to the composite structure as a whole and designates the resultative state of instrument in the action of cooking zuofan. Component Wo zuofan is viewed holistically and construed as a thing in the target structure. Horizontal correspondences constitute the relationship of integration, which links the component structure. The solid arrow leads from the e-site to its elaboration. Component Wo zuofan serves to elaborate the schematic trajector of the second verb zuo in Component zuohuai le guo. "Vertical" correspondences constitute the relationship of composition, which links the component structures to the composite structure. The solid arrow leads from the profile determinant to the composite structure.

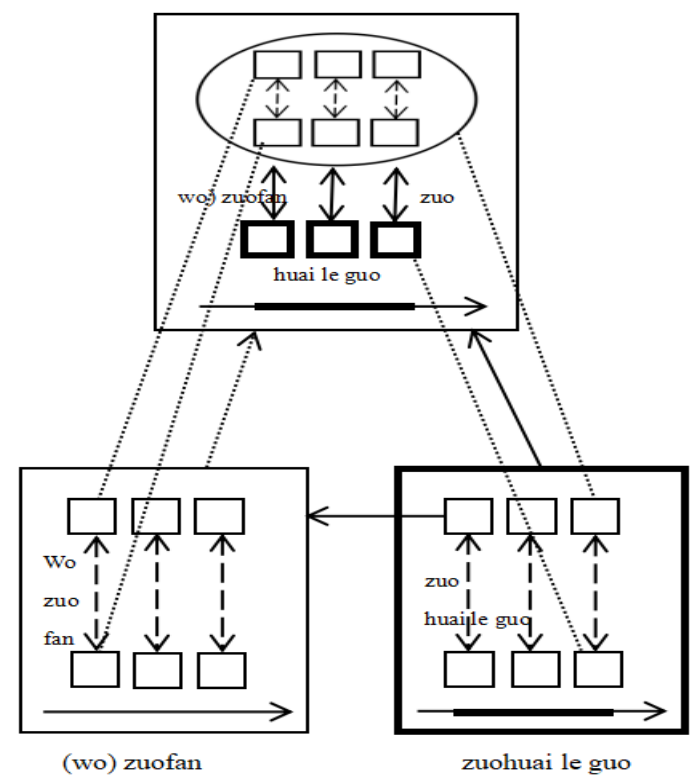

Figure 3. Compositional path of (Wo) zuofan zuohuai le guo 
The component structures constitute a symbolic assembly of complexity. Within this assembly, component structures serve as stepping-stones for arriving at composite structure, at two levels of organization. The ultimate target, shown at the top, comprises the composite form and meaning of the full expression. These stand in foreground. The path followed in reaching the final composite structure is a secondary but significant aspect of an expression's form and meaning.

The composite structure is not merely the sum of the component structures it is based on. It is an entity in its own right and has emergent properties not inherited or strictly predictable from the components and the correspondences between them. From Figure 3 alone, one could not predict that the composite expression Wo zuofan zuo huai le guo profiles the component VR zuohuai le guo rather than Wo zuofan. These are properties of the expression as a whole, emerging only at the composite expression. As a general matter, component structures should be thought of as resources drawn on-along with others-in arriving at the composite expression. While they motivate the composite structure to varying degrees, they should not be thought of as building blocks that need only be tacked together to form the composite whole. The relation between them is one of partial (rather than full compositionality) [22].

\section{MENTAL REPRESENTATION OF SVOVR}

CG emphasizes the psychological reality of linguistic representation. The representation of SVOVR construction fully corresponds to cognitive regularities of human beings. In this construction, component VR is the information focus and the profile determinant. The linguistic expressions are linear structures where the more specific first component provides potential for the second which is the conceptualization target and facilitates its emergence. This production process conforms to how we perform in the natural world. When we want to precisely describe the address of some places to a person, we may refer to another more well-known places or architectures to locate the target for the latter has more cognitive priority. Reference point is best described as the ability to invoke the conception of one entity for purpose of establishing mental contact with another, i.e., to single it out for individual conscious awareness [21]. The entity first invoked is called a reference point, and one accessed via a reference point is referred to as a target. A particular reference point affords potential access to many different targets. The salient reference point will pave the way for us to locate something. From the perspective of human cognitive processing, component VO lays the foundation for the whole conceptualization activity in VOVR construction.

In this construction, SVO has some kind of priority and provide substrate for the whole expression. This substrate allows the formation of more elaborate structures. In local terms, component SVO serves as the baseline which elaborates the trajector of the verb in component VR, forming a higher level $\mathrm{B}_{2}$. This higher level of baseline $\mathrm{B}_{2}$ is further elaborated by a Resultative complement, forming a higher level $\mathrm{B}_{3}$. Each component element is apprehended in relation to the one it directly follows. So moving from one stratum to the next, we encounter structures of greater complexity and conceptual sophistication [23]. SVOVR construction as a whole designates the resultative state of certain events. This cognitive processing, or operation, is completed in such order, which can be diagrammed as Figure 4. 


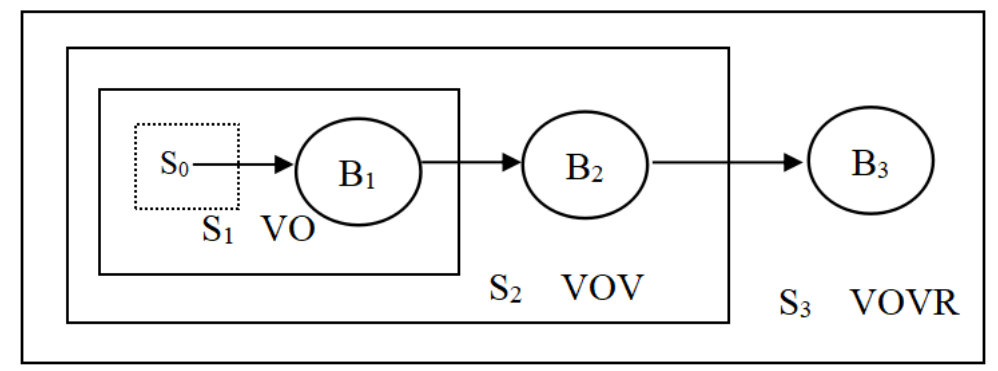

Figure 4. B/E organization of VOVR construction

SVOVR is a linear structure where VO evokes the baseline, also the reference point, and then the target VR can be accessible. The whole structure is scanned sequentially. The first baseline VO fades into the background after being evoked and the target VR comes to the foreground in a cumulative fashion. The mental representation of the SVOVR structure also corresponds to the properties that we access the target via the reference point. VO works as the reference point via which the conceptualizer establish mental contact with the conceptualization target, that is, the component VR. The reference point needs to be salient enough and thus can provide the substrate for the whole expression. By directing attention to a salient reference point to a salient reference point (R), the conceptualizer can readily access anything in the reference point's dominion (D), one such element being the target (T). This natural and efficient strategy is a basic feature of cognitive processing, evident in numerous aspects of linguistic structure [22]. The essential semantic import resides in the very act of mental scanning: evoking first the reference point and then a target it renders accessible. It is thus inherently and quintessentially dynamic, for how it unfolds through processing time actually constitutes its value (Langacker 2008: 84). Let's take the above sentences as example.

1. Wo zuofan zuo huai le guo My cooking broke the pan.

2. Wo zoulu zou de jiao qi pao le Walking made my feet blister.

3. Wo kanshu kan lei le Reading made me tired.

4. Wo jiao meishu jiao chu jingyan le Teaching art produced me some expertise.

In Example (1), VO zuofan provides the substrate and works as the baseline upon which the conceptualizer can access the target, i.e. the instrument in the event that VO designates. In (2), VO zoulu is the baseline and the body part of the agent involved in the action is the conceptualization target. In (3), paobu is the baseline and the whole body of the agent is the target for conceptualization. Likewise, jiao meishu is the baseline and the event as a whole is the target in (4). In these sentences, each stratum provides potential for the next, forming expressions with growing complexity.

Moreover, from a global perspective component SVO and VR function as dual baseline whose mutual elaboration yields a composite structure of greater complexity. The direction of composition, proceeding from "lower" to "higher" levels, is nothing other than the priority inherent in B/E organization, where each stratum provides the basis for arriving at the next [23]. The two 
component structure involves in different ways of operation from the perspective of $B / E$ organization. From "lower" to "higher" level, component SVO involves a process of modification whose profile changes from a processual relationship to a thing; component VR involves an operation of argumentation whose schematic trajector is specified by component SVO.

\section{RESTRICTIONS OF THE VERB IN SVOVR CONSTRUCTION}

SVOVR is a constructional schema abstracted from specific language use; it is a composite structure with SVO and VR as the component structure. In this construction, component VO provides the substrate and serves as the baseline based on which the conceptualization activity is completed in a cumulative fashion. Therefore, component VO needs to be substantive and have priority. There are some restrictions for verbs in SVOVR construction:

1. Component VO has more tendency to designate continuable and repeatable activities, which can be attributed to the fact that these activities can stimulate the following cognitive processing unfolded within the processing time. In other words, activities that are transient and unrepeatable like zhayan "blink" and tiaohe "drowning" cannot be trigger for the following conceptualization activity. Thus, verbal expressions that profile such activities have no occurrence in VOVR construction. Example (5) is ungrammatical because tiaohe "drowning" indicates the end of life according to our encyclopaedic knowledge. This event cannot be repeated and trigger the following conceptualization. On the contrary, tiaoshui "diving" is a kind of repeatable physical exercise. It can serve as the trigger for the following conceptualization activity.
i. $\quad *$ Tiaohe tiao lei le.
*Drowning makes sb tired.
ii. Tiaoshui tiao lei le.
Diving makes sb tired.

2. VO cannot be negated by the negative adverbials like $b u$ and mei for the negation means the termination of the following cognitive processing. Component SVO cannot serve as baseline once negated. Take Example (1) as an example, SVOVR will be ungrammatical if VO is negated. However, the negative adverbials can exist in component VR which means that the resultative state does not happen in the event.

- Zuofan zuo huai le guo.

- *Mei zuofan zuohuai le guo.

- Zuofan mei zuo huai guo.

3. The aspect markers like zhe/le/guo in Mandarin Chinese have no existence in Vo for the same reason as the previous one. VO has been reified as an object and serves as a reference point for the conceptualizer to access the target in its dominion. It rejects any aspect or tense markers of verbal expressions. Verb in VR structure profiles relationship and the VR structure designates the action results of SVO. In other words, despite the fact that there are two identical verbs in SVOVR construction, the two verbs have different meanings because of construal difference. The first verb profiles a kind of thing while the second one profiles a kind of relationship. As a reference point, VO structure will fade into the background and the 
target VR structure will be the figure. The whole composite structure will inherit the profile of VR and VR structure is the profile determinant of the composite structure.

Consequently, the traditionally accepted fact that verb copying is used to resolve the dilemma of object and adverbial fighting for the same verb is problematic from the standpoint of CG which claims that meaning is conceptualization; an expression's meaning is not just the conceptual content it evokes-equally important is how that content is construed [22]. Therefore, the two verbs in SVOVR construction have the same form with nonetheless different meanings; they function differently in the composite structure. Component SVO is construed as a thing and serves as a reference point while Component VR is target for conceptualization and profile determinant of the composite structure. In this construction, the repeated verb does not have the same meaning although it evokes the same conceptual content for the same verb is construed differently in the construction as a whole. In this sense, verb-copying is not for resolving the dilemma of object and adverbial competing for verb but a step-by-step statement of certain event and its result. On the other hand, the theory of competition is groundless due to the fact that in Chinese the first component can be Subject + Verb + Object and Subject + intransitive compound Verb as well. In the second case, it is impossible for object and adverbial competing for the same verb for there is no object.

\section{MOTIVATIONS OF SVOVR CONSTRUCTION}

According to CG, the grammar of a language can be characterized as a structured inventory of conventional linguistic units. The inventory is said to be structured in the sense that the units do not constitute encapsulated chunks of information; on the contrary, each unit stands at the hub of a network of relations to other units [20]. The inventory is structured in a sense that each established conventions participates in relations of various kinds to others. Doing CG consists, very largely, in elucidating these relations. It is these relations which, cumulatively, motivate a linguistic structure, in that they create a "niche" for the structure within the larger language system [20]. In other words, construction is unlikely to be represented in human mind without internal and external sanctioning conditions; these conditions motivate constructional representation both internally and externally. Therefore, SVOVR construction is not isolated from other facts about a language; its representation has both internal and external motivations. The conceptual overlap between the component structures, that is, the conceptual basis for the integration, is the internal motivation of the construction, which has been elaborated in Section 3. The external motivation gets its source from some established conventions in human's language system. In SVOVR construction, the two component structures are categorized by the established SVO and predicate-complement structures VR in Chinese language system. The two structures are schemas abstracted from linguistic expressions of various kinds, which have therefore been conferred on unit status in language system. Thus, SVOVR construction is more highly motivated than others due to the fact that its position in the language is supported by two highly established units.

\section{CONCLUSION}

This study unearths the cognitive mechanism of verb-copying sentences in Mandarin Chinese from the standpoint of CG; it investigates how the syntactic construction SVOVR is formed by the integration of component structure SVO and component VR. In this construction, SVO changes its profile from a processual relationship to a thing; the SVO structure elaborates the schematic trajector of structure VR in a specific and detailed way, which facilitates the integration of the two components into a composite structure. Component SVO designates the cause and component VR 
the effects; the latter component serves as the profile determinant of the composite structure. SVOVR construction involves both a serial and hierarchical B/E organization. Locally, each stratum provides potential for the next and thus forms the linear structure of growing complexity. In global terms, the two component structures serve as dual baselines whose mutual elaboration forms the composite whole. Conceptual correspondence of the component structures constitutes the internal motivation of the SVOVR construction; SVOVR construction is categorized by the conventionalized SVO and predicate-complement VR in Chinese language system, which forms the external motivation of SVOVR construction. This study has a different take on the traditional view that verbcopying serves to resolve the dilemma of object and adverbial competing for the same verb; it is an ordinal statement of an action and its result. This study contributes to the growing body of research on Chinese syntax and elucidates the relations each linguistic unit participate in with others.

\section{Acknowledgement}

This work is sponsored by the Postgraduate Research Innovation Project of Guangdong University of Foreign Studies "A Corpus-based Comparative Study of English and Chinese Human Metaphor in Economic Discourse” (Project No.: 19GWCXXM-03).

\section{Reference}

[1] Dai, H. Y. (1990). Yi renzhi wei jichu de hanyu gongneng yufa chuyi (Cognitive-based Chinese functional grammar). Guowai Yuyanxue (Foreign Linguistics), (4).

[2] Dai, H. Y. (1991). Yi renzhi wei jichu de hanyu gongneng yufa chuyi (Cognitive-based Chinese functional grammar). Guowai Yuyanxue (Foreign Linguistics), (1).

[3] Dai, Y. J. (1998). Shishuo hanyu chongdong ju de yufa jiazhi (Grammatical value of Chinese verb-copying sentences). Hanyu Xuexi (Chinese Language Learning), (2).

[4] Ding, C. C. (2015). Xiandai Hanyu Chongdong Ju de Renzhi Yanjiu (A Cognitive Study of Verb-copying Sentences in Modern Chinese). Ph.D Dissertation, East China Normal University.

[5] Li, L. D. (1980). Dongbu ge jushi (Verb-complement sentences). Zhongguo Yuwen (Studies of the Chinese Language), (2).

[6] Li \& Thompson (1981). Mandarin Chinese: A Functional Reference Grammar. University of California Press.

[7] Li, X. J. (2004). Chongdong ju de jizhong yuyong gongneng weitan (Exploring several pragmatic functions of verbcopying sentence. Sichuan Jiaoyu Хиеуuаn Xuebao (Journal of Sichuan Education University), (7).

[8] Liu, W. Q. (1986). Lun chongdong ju de tedian (Properties of verb-copying sentence), Nankai Daxue Xuebao (Journal of Nankai University),(3).

[9] Lu. J. M. (2013). Xiandai Hanyu Yufa Yanjiu Jiaocheng (Courses for Modern Chinese Grammar). Beijing: Beijing University Press.

[10] Nie, R. F. (2001). Chongdongju de yupian fenxi (Textual analysis for verb-copying sentence). Hunan Shifan Daxue Shehui Kexue Xuebao (Social Science Journal of Hunan Normal University), (1).

[11] Qin, L. J. (1985). Guanyu "dong+bin+dongchong+bu" de jiegou xingshi (Structural of "verb + object + copied verb + complement"), Yuyan Yanjiu (Linguistic Research), (2).

[12] Shen, J. X. (2005). Renzhi yu Hanyu Yufa Yanjiu (Cognition and Study of Chinese Grammar). Beijing: the Commercial Press.

[13] Tang, C. J. (2001). Xiandai hanyu chongdongju de fenlei (Classification of verb-copying sentence in modern Chinese). Shijie Hanyu Jiaoxue (Chinese Teaching in the World), (1).

[14] Wang, C. L. (1999). Chongdongju bu yi (Additional remarks on verb-copying sentences). Zhongguo Yuwen (Journal of Chinese Language), (2). 
Xu, Y. (2020). A Cognitive Linguistic Study of Verb-Copying Sentences in Mandrain Chinese. Advancees in Social Sciences Research Journal, 7(4) 164$\underline{176 .}$

[15] Wang, L. (1954). Zhongguo Yufa Lilun (Chinese Grammar Theory). Zhonghua Book Company.

[16] Xiang, K. X. (1997). Hanyu chongdong jushi de goneng yanjiu (Functional study of Chinese verb-copying sentence). Zhongguo Yuwen (Journal of Chinese Language), (4).

[17] Xiong, Z. R. (2017). Hanyu chongdongju de jufa fenxi (Syntactic analysis of Chinese verb-copying sentence). Huawen Jiaoxиe уи Yanjiu (TCSOL Studies), (2).

[18] Zhang, Y. (2018). "Ta de laoshi dang de hao" jushi: renzhi yufa shijiao. (Ta de laoshi dang de hao- perspective from cognitive grammar). Waiguo Yu (Journal of Foreign Language), (2).

[19] Zhao, Y. R. (1968). Grammar for Spoken Chinese. Beijing: the Commercial Press.

[20] Bybee, J. (2010). Language, Usage and Cognition. Cambridge: Cambridge University Press.

[21] Langacker, R. W. (1987). Foundations of Cognitive Grammar: Theoretical Prerequisites[M]. Stanford:Stanford University Press.

[22] Langacker, R. W. (2008). Cognitive Grammar: A Basic Introduction. New York: Oxford University Press.

[23] Langacker, R. W. (2016). Baseline and elaboration. Cognitive Linguistics, (27): 405-439. 